\title{
Testing critical point universality along the $\lambda$-line
}

\author{
J. A. Nissen ${ }^{1}$, D. R. Swanson ${ }^{1}$, Z. K. Geng ${ }^{1}$, V. Dohm², U. E. Israelsson ${ }^{3}$, \\ M. J. DiPirro ${ }^{4}$, and J. A. Lipa ${ }^{1}$ \\ ${ }^{1}$ Stanford University, Stanford, California 94305, USA \\ ${ }^{2}$ Institut fur Theoretische Physik, Technische Hochschule Aachen, D-52056 Aachen, Germany \\ ${ }^{3}$ Jet Propulsion Laboratory, California Institute of Technology, Pasadena, California 91109, USA \\ ${ }^{4}$ Goddard Space Flight Center, Greenbelt, Maryland 20771, USA \\ E-mail: joel@chex.stanford.edu
}

\begin{abstract}
We are currently building a prototype for a new test of critical-point universality at the lambda transition in ${ }^{4} \mathrm{He}$, which is to be performed in microgravity conditions. The flight experiment will measure the second-sound velocity as a function of temperature at pressures from 1 to 30 bars in the region close to the lambda line. The critical exponents and other parameters characterizing the behavior of the superfluid density will be determined from the measurements. The microgravity measurements will be quite extensive, probably taking 30 days to complete. In addition to the superfluid density, some measurements of the specific heat will be made using the low-g simulator at the Jet Propulsion Laboratory. The results of the superfluid density and specific heat measurements will be used to compare the asymptotic exponents and other universal aspects of the superfluid density with the theoretical predictions currently established by renormalization group techniques.
\end{abstract}

PACS: $64.60 . \mathrm{Ht}, \mathbf{6 7 . 4 0 . - w}$

\section{Introduction}

One of the major successes of the modern theory of continuous phase transitions is the calculation of exponents which characterize the singular behavior of thermodynamic properties near the transition temperature. Due to substantial theoretical difficulties, so far there exists no exact treatment of critical phenomena in three dimensions. Consequently the calculations of exponents are approximate in all cases of experimental interest. The renormalizationgroup (RG) [1] theory does, however, make a number of exact predictions concerning the nature of the critical exponents. One is that within broad limits the critical exponents are constants independent of microscopic interaction or of macroscopic thermodynamic parameters. They are said to be universal, depending only on the number of degrees of freedom of the order parameter and the number of spatial dimensions of the system. The transition from superfluid to normal fluid helium, the lambda transition, offers the best opportunity to verify this exact prediction with experiment. For example, near the lambda transition, the critical exponent $\zeta$ of the superfluid density, $\rho_{s}=\rho_{0}(P) t^{-\zeta}$, is predicted to be a universal constant close to $2 / 3$, where $t=1-T / T_{\lambda}(P)$ is the reduced temperature measuring the distance from the transition temperature, $T_{\lambda}$. By contrast, the amplitude $\rho_{0}(P)$ is a nonuniversal quantity depending on the pressure, $P$, of the isobar on which $\rho_{s}(t)$ is measured. Along with the critical exponents, certain ratios of amplitudes of thermodynamic quantities are also predicted to be universal [2]. Most data are roughly consistent with the predicted universality, i.e. with constant values of the critical exponents and amplitude ratios. However, in virtually all systems of interest the level of testing is very weak. Even in the case of the lambda transition of helium, where the most advanced tests have been performed [3], some anomalies have been detected [4].

Unlike continuous transitions in other fluids, the lambda transition is not a single critical point but rather is a line of transitions obtained by varying the hydrostatic pressure. It is possible to measure the critical exponent of the superfluid density with exceptional ease and accuracy due to a unique wave motion termed second sound, in which the temperature of the liquid rather than its pressure oscillates. 
Over the years many measurements of the velocity of second sound have been made, but only recently have attempts been made to access the deep asymptotic region, close to the transition, where the theoretical predictions are most accurate. Now it is possible to operate with the reduced temperature as small as $10^{-8}$, a factor of 1000 less than in earlier experiments. Previous ground-based experiments [5] indicate that the critical exponent for the superfluid density can be measured to better than 1 part in $10^{4}$. This is about an order of magnitude better than is possible with any other exponent, for any known physical system. With care, perhaps even higher accuracy can be obtained.

Unfortunately, the presence of gravity acting on the experimental cell reduces the reliability of the analysis of the results. The effects of gravity can be cancelled to first order by measuring the temperature $T_{\lambda}$ at the middle of the cell, however, close to the transition, gravity plays a dominant role in determining the apparent velocity of the wave, even in small cells. For $t \leqslant 10^{-7}$ second-order effects become important. Specifically the term $\nabla \rho_{s}$ must be included in the differential equation describing second sound [6]. Further reduction in cell size, below about $1 \mathrm{~mm}$ height, is inhibited by the presence of finite size effects which distort the singular behavior of interest. To obtain a more accurate reflection of the idealized fluid behavior it is necessary to substantially reduce the effect of gravity in the experiment by performing the experiment in microgravity conditions.

Great care must be exercised in the interpretation of such experiments. At higher pressures, nonasymptotic corrections to the superfluid density become more important, leading to an effective exponent which varies with pressure [7]. Clearly it is very important to distinguish between this nonuniversal effect and a violation of universality. Fortunately, a correction scheme has been developed [8], based on RG calculations, which can be used to separate the non-universal from the universal effects in a quantitative way. Its utility has already been demonstrated on existing data over a restricted temperature range [9]. Part of the project will include a theoretical effort to improve the accuracy of this approach and define its limits more clearly

To apply the correction, supplementary measurements of specific heat, $C_{p}$, or thermal expansion, $\beta_{p}$, are needed at all pressures of interest. Measurements performed in the Jet Propulsion Laboratory's low-g simulator of the expansivity at various pressures will provide the needed data for the correction terms to the asymptotic behavior. If a pressure dependence of the superfluid density exponent along the lambda line is established, this would constitute a substantial problem for condensed matter theory: one of the central results of the RG theory on which the modern view of phase transitions is built would have been violated. Agreement with theory would represent the strongest support yet obtained.

\section{Universality}

One of the major achievements of the RG theory [1] was the proof of the universality hypothesis [10] of the critical behavior and the specification of the characteristics of the universality classes. Critical points of different physical systems having the same dimensionality and the same number of degrees of freedom of the order parameter are predicted to belong to the same universality class with identical critical exponents, scaling functions and certain ratios of amplitudes of singular thermodynamic quantities [2]. These universal properties are predicted to be independent of the strength of the interaction and can be calculated by RG methods on the basis of a statistical distribution $p(\Psi)$ for the order parameter $\Psi$. For example, the lambda transition of ${ }^{4} \mathrm{He}$ is governed by the two-component (complex) wave function $\Psi$ of the Bose condensate and should have universal properties that are identical with those of two-component spin systems. In particular, these properties should be independent of the pressure $P$ at which the lambda transition occurs. This prediction can be tested by measuring thermodynamic quantities such as the superfluid density $\rho_{s}$, the specific heat $C_{p}$, and the thermal expansion coefficient $\beta_{p}$. The latter is known [3] to exhibit the same critical behavior as $C_{p}$. In a highly quantitative analysis of critical phenomena, nonasymptotic corrections are inevitable because data must be taken a finite distance from the transition temperature. The amplitudes of the correction terms are predicted to be nonuniversal and are expected to increase with increasing pressure $[8,11]$. Thus the test of universality in the high-pressure region will be planned at the outset as an analysis including non asymptotic correction terms. These terms contain both universal and nonuniversal parameters. More specifically, close to the transition temperature, $T_{\lambda}(P)$, RG theory predicts [12] that

$$
\rho_{s}=\rho_{0}|t|^{v}\left(1+a_{\rho}|t|^{\Delta}+\ldots\right)
$$

where the critical exponent for the superfluid density, $\zeta$, can be shown to be equal to the critical 
exponent for the correlation length, $v$, by the Josephson relationship. Also,

$$
C_{p}=B+\frac{A^{ \pm}}{\alpha}|t|^{-\alpha}\left(1+a_{c}^{ \pm}|t|^{\Delta}+\ldots\right)
$$

where $\alpha$ is the critical exponent for the heat capacity at constant pressure and the \pm signs refer to $T>T_{\lambda}$ or $T<T_{\lambda}$, respectively. Within the RG calculation the quantities $\rho_{0}, B, A^{+}, A^{-}$, as well as the correction amplitudes $a_{\rho}, a_{c}^{+}, a_{c}^{-}$turn out to depend on the (pressure dependent) parameters of the statistical distribution $p(\Psi)$ whereas the critical exponents $v, \alpha$ and the correction exponent $\Delta$ are universal, i.e., independent of these parameters. Certain ratios of the nonuniversal amplitudes, however, are predicted to be universal as well, for example, $A^{+} / A^{-}, a_{c}^{+} / a_{c}^{-}, a_{\rho} / a_{c}^{-}$. The analysis consists of two steps: first, to extract from the data those quantities that are predicted to be universal, and to determine their pressure dependence; second, to compare the measured values of these quantities with those predicted by RG calculations. Such analyses have previously been performed [3] for the superfluid transition of ${ }^{4} \mathrm{He}$ at a level of quality limited by the accuracy of the data $[7,13]$ available about 20 years ago.

The representations of $\rho_{s}$ and $C_{p}$ according to (1) and (2) are approximate in the sense that there exists a whole series (Wegner series [14]) of additional correction terms of the form $t^{2 \Delta}$, $t^{3 \Delta}$, etc. along with other terms. An appropriate application of the RG method makes it possible to sum up this Wegner series and to represent the correction terms in a closed functional form $[8,9,15]$. This representation is more complete and more accurate than the standard form (1) and (2) while containing the same number of nonuniversal parameters. It includes accurate field-theoretic functions obtained via high-order perturbation theory and Borel resummation [16]. Part of the planned theoretical effort is to calculate not only the universal amplitude ratios but also the resummed functional form of the correction terms more accurately by means of the field-theoretic RG approach. This will provide a more reliable basis for the combined non-asymptotic analyses of the data for $\rho_{s}$ and $C_{p}$ (or $\beta_{p}$ ) and will allow one to perform a controlled analysis over a larger temperature range outside the small asymptotic region. Furthermore it is planned to study the effect of additional correction terms arising from a possible cut-off dependence of the statistical distribution $p(\Psi)$.

\section{Second sound}

The central part of the experiment is a cylindrical resonator filled with high purity liquid helium. The sample chamber forms a second-sound resonator with a quality factor, $Q$, typically over 1000 . The $Q$ is limited by losses in the fluid and the end walls. Second-sound detectors based on rf squid technology with sensitivities of less than $10^{-10} \mathrm{~K} / \sqrt{\mathrm{Hz}}$ are already in routine laboratory use. For a flight experiment the maximum signal/noise ratio is desirable to decrease the measuring time. For this reason the sensing element will be coupled to an improved sensitivity dc squid magnetometer. Also to be evaluated is the two-stage SQUID readout scheme being developed at Goddard, which promises even lower noise [17]. The second-sound resonator will utilize a magnetically driven paramagnetic salt as a low dissipation second-sound generator.

The resonator is attached to a high resolution paramagnetic salt thermometer and a superconducting pressure controller. The proposed experiment makes full use of the high resolution thermometry [18] developed primarily for the Lambda Point Experiment (LPE) [19] and the Confined Helium Experiment (CHEX) [20] flight programs. The present status of the high resolution thermometry is that the noise level is set by intrinsic thermal fluctuation phenomena. In the CHEX configuration this leads to a limiting resolution of about $10^{-10} \mathrm{~K}$ in a $1 \mathrm{~Hz}$ bandwidth. Integration over longer periods allows resolutions deep in the $10^{-12}$ range with the ultimate performance set by sensor and electronics drift. The pressure transducer is currently under development with separate NASA funding and has not yet been flown. It represents a significant development effort, but all elements of the device exist in other applications. The main parts of the sensor element are derived from the gravitational wave detector at Stanford where displacements of $10^{-17} \mathrm{~m}$ are monitored. This demonstrated sensitivity far exceeds our needs. Closed loop pressure control at resolutions of $10^{-9}$ has not yet been demonstrated,

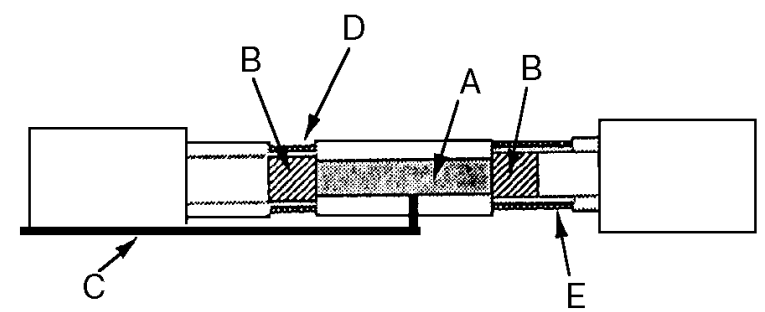

Fig. 1. Second-sound resonator : A) helium, B) paramagnetic salt, C) fill line, D) pickup coil, E) driving coil and astatic coil. 
but bandwidth requirements are extremely modest, less than $1 \mathrm{~Hz}$, so little difficulty is expected.

This assembly will be placed within a multistage thermal enclosure similar to that flown on the LPE. The only significant change in this part of the apparatus will be the use of a tighter thermal link between the cell and the inner stage of thermal isolation. This change will allow a faster response thermal control loop on the cell, and more rapid cooling for repeating temperature scans. The walls of the enclosure will be maintained near $1.7 \mathrm{~K}$ to allow operation of the sample chamber at various temperatures along the lambda line. This basic operating temperature will be maintained by using a superfluid helium cryostat with a long hold time (30-50 days) which is needed for completing the measurements at a number of different pressures.

To perform the experiment, the pressure is first set at the desired value by adjusting the reference pressure of the controller system using high pressure ${ }^{3} \mathrm{He}$ gas. This level of pressure adjustment need only be done relatively crudely, say to an accuracy of about $1 \%$. Once set, this pressure needs to be held constant to within about 1 part in $10^{9}$ using the internal controller. Next, the temperature of the cell is raised ever closer to the lambda point and the fundamental resonant frequency plus harmonics of the cavity are measured. Values in the range 1 to $500 \mathrm{~Hz}$ are expected. Frequency and temperature measurements are continued until the transition is reached, marked by loss of the signal. A number of temperature sweeps at a given pressure will be performed to check for errors in the data and to estimate the uncertainties in the results. Additional checks on the transition temperature will be made by observing the response of the cell to pulsed heat inputs applied with the second-sound generator. The pressure would then be reset and the measurement sequence repeated. In each resonant frequency measurement, the $Q$-curve of the resonator would be scanned to improve the accuracy of the determination of the central frequency.

It should be noted that second-sound measurements have already been made to within $20 \mathrm{nK}$ of the transition in ground experiments [5,21]. Thus there is no additional need to demonstrate feasibility of the basic measurement technique. The problem is to reduce the huge gravitational distortion that makes such experiments difficult to interpret. Only a layer of the sample about $0.2 \mathrm{~mm}$ thick can be considered to be that close to the transition; the rest is further away due to the shift in $T_{\lambda}(P)$ caused by the hydrostatic head. In space, the effective thickness of this layer will grow to about $10 \mathrm{~cm}$, filling the whole resonator. This measurement would also give new information on a second parameter of interest, the damping coefficient of the thermal wave. The behavior of this quantity near the transition is of importance in the testing of the theory of dynamic critical phenomena.

\section{Expansivity}

To perform the tests of the universality prediction for the superfluid density it is necessary to have information on the heat capacity behavior (or $\beta_{p}$ ) over a limited range. We plan to use the low-g simulator at JPL for these measurements. The volume over which the effective gravity environment can be reduced to the $0.01 \mathrm{~g}$ level in the simulator is limited to about 0.5 cubic centimeter. Due to the necessarily small sample volume we believe the highest quality data can be obtained by performing expansion coefficient measurements, which relate directly to heat capacity data. Following Mueller et al. [13], the expansion coefficient measurements will be performed using a standard «hot volume» technique in which the sample helium in the lowgravity simulator is connected via a small capillary to a second volume containing a high resolution pressure gauge. The temperature of the hot volume chamber is changed in response to a small change in the sample volume temperature such that the pressure is held constant. The expansion coefficient is calculated from the applied change in temperature in the sample cell and the calculated change in density of the hot volume as deduced from it's change in volume. A pressure regulation of 0.15 mbar has been demonstrated routinely with this technique corresponding to a temperature noise in the sample chamber of about $0.3 \mathrm{nK}$ at a pressure of $10 \mathrm{bar}$ and $3 \mathrm{nK}$ at a pressure of 1 bar. We plan to take data from 1 bar up to 30 bar. Due to the very large magnetic field present in the low-g simulator, temperatures are measured using melting curve thermometry [22]. The demonstrated resolution of these thermometers is about $3 \mathrm{nK}$ in a $1 \mathrm{~Hz}$ bandwidth. The proposed measurement technique would thus be limited by thermometer noise in all but the very lowest pressure range studied.

\section{Conclusion}

The results from the experiment described here would become a major new test of the theory of second-order phase transitions in condensed matter. The measurements would probe the applicability of the universality prediction to an unprecedented degree, and possibly to the maximum extent feasible 
with known technology. If the prediction is found to fail, it would be a major blow to current ideas on the origins of second-order transitions, and would mean that critical phenomena are more complicated than the RG theory suggests. If it is found to be valid, this theoretical framework, for which K. Wilson received the Nobel Prize in 1982, would be placed on a much sounder footing of supporting experimental evidence. As a secondary result, new data would be obtained on the behavior of the second sound damping coefficient very near the transition, as an aid to the developing theory of dynamic critical phenomena. The experiment is expected to be available for launch in the second half of 2002 .

\section{Acknowledgements}

We wish to thank the Office of Life and Microgravity Science and Applications of NASA for its support with contract JPL 960613.

1. K. G. Wilson and J. Kogut, Phys. Rep. C12, 76 (1974); M. E. Fisher, Rev. Mod. Phys, 46, 597 (1974).

2. V. Privman, P. C. Hohenberg, and A. Aharony, in: Phase Transitions and Critical Phenomena 14, C. Domb and J. Lebowitz (eds. ), Academic Press, NY (1991), p. 1.

3. A. Singsaas and G. Ahlers, Phys. Rev. B30, 5103 (1984); G. Ahlers, Rev. Mod. Phys. 52, 482 (1980); T. Takada and T. Watanabe, J. Low Temp. Phys. 41, 221 (1980); F. M. Gasparini and M. R. Moldover, Phys. Rev. B12, 93 (1975); also, Ref. 12.
4. F. M. Gasparini and A. A. Gaeta, Phys, Rev, B12, 1466 (1978); see also: J. A. Lipa, Q. Li, T. C. P. Chui, and D. Marek, Nucl. Phys. B, 5A, 31 (1988).

5. M. J. Adriaans, D. R. Swanson, and J. A. Lipa, Physica 194-196, 733 (1994).

6. D. R. Swanson, T. C. P. Chui, and J. A. Lipa, Phys. Rev. B46, 9043 (1992).

7. D. S. Greywall and G. Ahlers, Phys, Rev. A7, 2145 (1973).

8. V. Dohm, Z. Phys. B60, 61 (1985); J. Low Temp. Phys. 69, 51 (1987).

9. R. Schloms and V. Dohm, Europhys. Lett. 3, 413 (1987).

10. L. P. Kadanoff, in: Critical Phenomena, Proc. of the Enrico Fermi Int. School of Physics 51, M. S. Green (ed.), Academic Press, New York (1971), p. 100.

11. V. Dohm, in: Proc. of the 17th Int. Conf. on Low Temp. Phys, U. Eckern, A. Schmid, W. Weber, and H. Wuehl (eds.), North Holland (1984), p. 953.

12. R. Schloms and V. Dohm, Phys. Rev. B42, 6142 (1990).

13. K. H. Mueller, G. Ahlers, and F. Pobell, Phys. Rev. B14, 2096 (1976).

14. F. Wegner, Phys. Rev. B5, 4529 (1972).

15. V. Dohm and R. Schloms, unpublished.

16. R. Schloms and V. Dohm, Nucl. Phys. B328, 639 (1989).

17. M. DiPirro, P. J. Shirron, and J. G. Tuttle, Proc. 1994 Microgravity Low Temp. Phys. Workshop, p. 374 (1994).

18. T. C. P. Chui, D. Swanson, M. J. Adriaans, J. A. Nissen, and J. A. Lipa, Phys. Rev. Lett. 69, 3005 (1992).

19. J. A. Lipa, D. R. Swanson, J. A. Nissen, T. C. P. Chui, and U. E. Israelsson, Phys. Rev. Lett. 76, 944 (1996).

20. X. Qin, J. A. Nissen, D. R. Swanson, P. R. Williamson, D. A. Stricker, J. A. Lipa, T. C. P. Chui, and U. E. Israelsson, Cryogenics 36, 781 (1996).

21. M. J. Adriaans, Thesis, Stanford University (1994).

22. L. S. Goldner, N. Mulders, and G. Ahlers, Proc. Int. Symp. Temp. (1992). 